\title{
NUEVOS TAXA EN GRAMMITIDACEAE (PTERIDOPHYTA) DE COSTA RICA
}

\author{
Alexander Fco. Rojas Alvarado \\ Jardín Botánico Lankester, Universidad de Costa Rica, \\ apdo.1031-7050, Cartago, Costa Rica. afrojasa@yahoo.com
}

\begin{abstract}
Lellingeria pinnata A. Rojas and Terpsichore glandulifera A. Rojas are described as new to science, illustrated, and compared to the related species L. phlegmaria (J.Sm.) A.R. Sm. \& R.C. Moran and T. turrialbae (H. Christ) A.R. Sm., respectively. Terpsichore pirrensis A.R. Sm. is here first reported from Costa Rica.
\end{abstract}

Resumen. Lellingeria pinnata A. Rojas y Terpsichore glandulifera A. Rojas se describen, se ilustran y se comparan con las especies cercanas L. phlegmaria (J.Sm.) A.R. Sm. \& R.C. Moran y T. turrialbae (H. Christ) A.R. Sm., respectivamente. Terpsichore pirrensis A.R. Sm. es un nuevo registro de Costa Rica.

Palabras clave / Key words: Pteridophyta, Grammitidaceae, Lellingeria, Terpsichore, Costa Rica.

El género Lellingeria fue descrito por Smith et al. (1991) y se diferencia de otros géneros de Polypodiaceae (grupo Grammitidae) por tener un rizoma simétricamente radial, pelos desigualmente furcados y escamas del rizoma clatradas.

El género Terpsichore fue descrito por Smith (1993) y caracterizado por la presencia de hidátodos conspicuos (que algunas veces producen secreciones calcáreas), setas rojizas a atropurpúreas (raro hialinas) de 1-3 mm de largo en pecíolo, raquis, costa y algunas veces en la lámina, escamas del rizoma no clatradas y usualmente castañas a negruzcas, que a menudo son setulosas en el margen, y venas libres, no ramificadas y pinnadas en cada pinna.

Las especies de Lellingeria descritas de Costa Rica en las últimas tres décadas son: L. barbensis (Lellinger) A.R. Sm. \& R.C. Moran (= Grammitis barbensis Lellinger), L. melanotrichia (Baker) A.R. Sm. \& R.C. Moran (= G. micula Lellinger) y $L$. pseudomichellae (Lellinger) A.R. Sm. \& R.C. Moran (= G. pseudomichellae Lellinger) (Lellinger 1985). Del género Terpsichore se han descrito de Costa Rica recientemente: T. zeledoniana (Lellinger) A.R. Sm. (= Grammitis zeledoniana Lellinger) (Lellinger 1985), T. cocoensis A. Rojas (Rojas 1996) y T. esquiveliana A. Rojas (Rojas 2001).

\section{DOS ESPECIES NUEVAS}

\section{Lellingeria pinnata A. Rojas, sp. nova}

A Lellingeria phlegmaria (J. Sm.) A.R. Sm. affinis, sed lamina pinnata, rachidi flexuosa, distributione

\section{altitudinali inferiore distinguenda.}

TIPO: COSTA RICA. Limón; Limón, fila Matama, valle de La Estrella, El Progreso, siguiendo la fila a

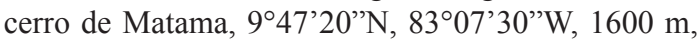
23 abr 1989, G. Herrera \& A. Chacón 2728 (Holotipo: $\mathrm{CR}$, isotipos: $\mathrm{K}, \mathrm{MO})$. Fig. 1.

Epífita; rizoma de 1-2 mm de diámetro, compacto; escamas del rizoma de 1.5-2.5 x mm, lanceoladas, pardo-rojizas, setulosas; pecíolo $0-0.5 \mathrm{~cm}$, setuloso, las sétulas ca. $0.2 \mathrm{~mm}$, hialinas; lámina (3-)5-20 x 1-2 cm, pinnatisecta en toda su longitud, linear o angostamente oblonga, ligeramente atenuada hacia la base, esparcidamente setulosa en la costa y los márgenes, glabra entre éstos; segmentos $2-3 \mathrm{~mm}$ de ancho en la base, enteros o con 1 ó 2 grandes crenas en cada lado, ligeramente ascendentes; raquis recto (no flexuoso), setuloso, las sétulas $c a .0 .2 \mathrm{~mm}$, hialinas, a menudo ramificadas; nervaduras fértiles no visibles; hidátodos 6-14 por segmento fértil; soros redondeados, no hundidos o ligeramente impresos; cápsulas esporangiales glabras.

Distribución. Hasta ahora sólo conocida del Volcán Cacao en la Cordillera de Guanacaste y la fila Matama en la Cordillera de Talamanca, Costa Rica, entre 1300 y $1600 \mathrm{~m}$.

Paratipo: COSTA RICA. Guanacaste; Liberia, Parque Nacional Guanacaste, Volcán Cacao, entre la ramificación del sendero a la Estación Maritza y la punta del cerro, $10^{\circ} 56^{\prime} 08^{\prime \prime} \mathrm{N}, 85^{\circ} 27^{\prime} 10^{\prime \prime} \mathrm{W}, 1300-1600$ 
m, 5 febr 1998, A. Rojas \& A. Soto 4257 (CR, INB).

Lellingeria pinnata difiere de L. phlegmaria por la lámina pinnada (vs. pinnatisecta), el raquis negro (vs. del color de la lámina), lustroso (vs. opaco) y flexuoso (vs. recto), la base de las pinnas distantes entre sí 1-3 $\mathrm{mm}$ (vs. base decurrente hasta el segmento anterior), el margen de los segmentos entero (vs. ondulado a crenado) y la distribución a menor elevación (1300$1600 \mathrm{~m}$ vs. $2000-2800 \mathrm{~m}$ ).

Etimología. El epíteto pinnata de esta especie se refiere a la lámina foliar pinnada, carácter muy raro en este género.

\section{Terpsichore glandulifera A. Rojas, sp. nova}

A Terpsichori turrialbae similis, sed frondibus longioribus, lamina abaxialiter dense setosa, pilis glanduliferis lacteis vel flavidis, sporangiis setulosis, distributione altitudinali inferiore recedit.

TIPO: COSTA RICA. Cartago: Paraíso, Parque Nacional Tapantí, cuenca del Río Reventazón, Orosi, sendero Palmitos o T6, 9 $43^{\prime} 35^{\prime \prime} \mathrm{N}, 8^{\circ} 46^{\prime} 28^{\prime \prime} \mathrm{W}$, 1460-1600 m, 3 sept 1997, A. Rojas \& R. Delgado 3788 (Holotipo: CR, isotipos: INB, MO). Fig. 2.

Epífita; rizoma 1-2 mm de diámetro; escamas del rizoma ausentes; hojas péndulas, el ápice de crecimiento indeterminado; pecíolo $1-3 \mathrm{~cm} \times 0.4-0.6 \mathrm{~mm}$, pardo claro a oscuro, las setas 2-3 mm, numerosas, hialinas a rojizo pálidas; lámina 15-75(-100) x (2.5-)3-6(-8) $\mathrm{cm}$, pinnada o pinnatisecta, linear a angostamente oblanceolada, gradualmente reducida en la base, setosa y puberulenta, generalmente con numerosos tricomas glandulares, sin hongos claviformes negros; raquis pardo a negro, setoso y puberulento, las setas $1-2 \mathrm{~mm}$, no pareadas, rojizo pálido a hialinas, los tricomas 0.1$0.2 \mathrm{~mm}$, ramificados o simples, a menudo glandulares, rojizo pálido; pinnas (1.3-)1.5-4 x 0.2-0.5 cm, casi perpendiculares al raquis o ligeramente ascendentes, adnatos $c a .2 / 3$ de su ancho, distantes entre sí más de su ancho en la base, la base con el lado basiscópico truncado o a menudo con un lóbulo sub-basal, raro sólo levemente decurrente, los márgenes setosos, el ápice agudo a obtuso, las setas $0.5-2 \mathrm{~mm}$, no pareadas, pareadas o raramente sésil-estrelladas, hialinas a rojizo pálidas; costas y nervaduras inconspicuas o negras y evidentes, con indumento similar al del raquis $\mathrm{y}$ generalmente con numerosos tricomas glandulosos hialinos a amarillentos; hidátodos con puntos blanquecinos calizos; soros setosos desde los receptáculos o no setosos; esporangios setosos, los tricomas $0.5-1.0 \mathrm{~mm}$ de largo, no glandulares y de ápice agudo.

Distribución. Hasta ahora sólo conocida de la Cordillera de Tilarán, Cordillera Central y Cordillera de Talamanca en Costa Rica; 1400-2000 (-2300) m.

PARATIPOS: COSTA RICA. Braulio Carrillo National Park, 19 jul 1993, E. Schmid 161 (CR). Alajuela: San Ramón, Reserva Biológica Monteverde, Cordillera de Tilarán, valle del Río Peñas Blancas, $10^{\circ} 19^{\prime} 15^{\prime} \mathrm{N}$, 8446’30”W, 1600 m, 22 jul 1993, E. Bello 5158 (INB, CR, MO); Monteverde Forest Reserve, Sendero Río and Sendero Chomogo, $10^{\circ} 15^{\prime} \mathrm{N}, 84^{\circ} 50^{\prime} \mathrm{W}, 1575 \mathrm{~m}, 31$ oct 1986, E. Hennipman et al. 6576 (CR). AlajuelaHeredia: $100 \mathrm{~m} \mathrm{~N}$ del Restaurant Vara Blanca, $1920 \mathrm{~m}$, 4 febr 1966, A. Jiménez 3670 (CR). Cartago: Paraíso, slopes and flats at $\mathrm{S}$ end of bridge over río Grande de Orosi, Tapantí, $9^{\circ} 46^{\prime} \mathrm{N}, 83^{\circ} 48^{\prime} \mathrm{W}, 1200 \mathrm{~m}, 24$ oct 1989 , M. Grayum et al. 9526 (CR); Paraíso, cuenca del Río Reventazón, Estación de Biología Tropical Río Macho y alrededores, $9^{\circ} 46^{\prime} \mathrm{N}, 83^{\circ} 51^{\prime} \mathrm{W}, 1630-1730 \mathrm{~m}, 27$ sept 1996, A. Rojas et al. 3115 (CR, INB, K, MO, UC, US); Paraíso, Parque Nacional Tapantí, Cuenca del río Reventazón, camino entre las torres, desde los nacientes del Río Grande de Orosi a Rancho Negro, 941'34”N, 8347'11'”, 1700-2300 m, 23 mayo 1997, A. Rojas et al. 3494 (CR, INB); along trail leading eastward into mountains from road into Tapantí Reserve, $c a .1 \mathrm{~km} \mathrm{~S}$ of junction of quebrada Salto and Río Grande de Orosi, also known as “Quebrada Valverde", $9^{\circ} 43^{\prime} \mathrm{N}, 83^{\circ} 47^{\prime} \mathrm{W}$, 1500-1800 m, 1 febr 1986, A. Smith et al. 2154 (CR, UC). Heredia: Heredia, Cordillera Central, Vara Blanca, camino al Volcán Poás y rumbo a Cariblanco, $10^{\circ} 09^{\prime} 35^{\prime \prime} \mathrm{N}, \quad 84^{\circ} 09^{\prime} 25^{\prime \prime} \mathrm{W}, 1850-1930 \mathrm{~m}, 2$ mar 1995, A. Rojas \& M. Piepenbring 1718 (CR); Finca of Dr. L. Holdridge, on the Río Puerto Viejo, near the junction with the Sarapiquí, $c a .300 \mathrm{ft}$. [90 m], 18-28 febr 1955, E. Scamman 7519 (CR); Cascajal, 1800 m, nov 1978, Wagner \& L. Gómez 78534 (CR). Limón: Cordillera de Talamanca, Atlantic slope, canyon of the Río Siní, 9¹3’N, 8259’W, 1800-1900 m, 15 sept 1984, G. Davidse \& G. Herrera 29145 (CR, MO). Puntarenas: Sitio Cotón, base SW de Cerro Pando, 1300 m, febr 1982, L. Gómez 18140 (CR). San José: between Bajo La Hondura and Alto La Palma, $10^{\circ} 2^{\prime} \mathrm{N}$, 8359'W, 1400-1500 m, 19 jul 1983, K. Barringer et al. 3947 (CR). Parque Nacional Braulio Carrillo, Cordillera Central, along ridge to $\mathrm{W}$ of Tunel Zurquí, along headwaters of Río Hondura, E slopes of Cerro 
Hondura, $10^{\circ} 04^{\prime} \mathrm{N}, 84^{\circ} 1.5^{\prime} \mathrm{W}, 1600-1800 \mathrm{~m}, 18$ mayo 1989, M. Grayum et al. 9487 (CR, MO).

La nueva especie difiere de Terpsichore turrialbae por las frondas relativamente más largas $(15-100 \mathrm{~cm}$ vs. $10-45 \mathrm{~cm}$ de largo), las pinnas distantes entre sí más de su ancho (vs. pinnas distantes menos de su ancho), la base de las pinnas con el lado basiscópico truncado, a menudo con un lóbulo sub-basal, raro sólo levemente decurrente (vs. base de las pinnas decurrente), las glándulas laminares hialinas a amarillentas (vs. pardas a pardo-rojizas), los esporangios setosos, los tricomas 0.5-1.0 mm (vs. glabros o con tricomas capitados de menos de $0.1 \mathrm{~mm}$ ) y la distribución altitudinal a $1400-$ 2000 (-2300) m [vs. 2400-2900 m].

Etimología. El nombre de esta especie, T. glandulifera, se refiere a los abundantes pelos glandulares en el envés de la lámina.

Nuevo Registro en Costa Rica

Terpsichore pirrensis A.R. Sm., Novon 3: 483. 1993.

Tipo: Panamá, Darién, Cerro Pirre, 2500-4500ft. [7601370 m], Duke \& Elias 13842 (Holotipo: UC, no visto; Isotipos: MO!, NY!, SCZ).

Características muy particulares, como los tricomas cortos en el pecíolo, hacen a esta especie inconfundible dentro del género (Fig. 3).

\section{Distribución General. Costa Rica y Panamá.}

Especímenes observados: COSTA RICA. Heredia: along the Río Segundo, $2 \mathrm{~km}$ of Cerro Chompipe, $8 \mathrm{~km}$ NNE of Heredia, $1900 \mathrm{~m}, 12$ jul 1970, D. Lellinger \& J. White 1089 (CR). Puntarenas: Coto
Brus, Zona Protectora Las Tablas, camino a Cerro Chai, Las Alturas, $8^{\circ} 58^{\prime} 00^{\prime \prime} \mathrm{N}, 82^{\circ} 49^{\prime} 40^{\prime \prime} \mathrm{W}, 2000 \mathrm{~m}$, 4 sept 1992, A. Fernández 375 (INB, MO); Coto Brus, Zona Protectora Las Tablas, Cordillera de Talamanca, Sabalito, Las Alturas de Cotón, Estación Biológica Las Alturas, sendero a Cerro Echandi, postes 40-57, $8^{\circ} 59^{\prime} 05^{\prime} \mathrm{N}, 82^{\circ} 43^{\prime} 10^{\prime \prime} \mathrm{W}, 2070-2230 \mathrm{~m}, 28$ dic 1993 , A. Rojas 822 (INB); Coto Brus, Zona Protectora Las Tablas, cuenca Térraba-Sierpe, camino a Quijada del Diablo, 8०54'21”N, 8245'04”W, 2100 m, 20 nov 1996, A. Rojas et al. 3298 (CR, INB); Parque Internacional La Amistad, La Amistad, Cafrosa, $8^{\circ} 54^{\prime} 40^{\prime \prime} \mathrm{N}, 82^{\circ} 47^{\prime} 19^{\prime \prime} \mathrm{W}, 2000 \mathrm{~m}, 19$ jun 1990, $J$. Saborio et al. 43 (CR).

Agradecimientos. Agradezco a los herbarios del Museo Nacional de Costa Rica (CR), Instituto Nacional de Biodiversidad (INB) y University of California (UC) por permitirme utilizar sus colecciones, a Franco Pupulin por las diagnosis latinas y a los revisores anónimos por sus comentarios, que mejoraron la calidad de este estudio.

\section{LiterATURA CITADA}

Lellinger, D.B. 1985. Nomenclatural and taxonomic notes on the Pteridophytes of Costa Rica, Panama and Colombia, II. Proc. Biol. Soc. Wash. 98: 366-390.

Rojas, A.F. 1996. Aportes a la Flora Pteridophyta Costarricense. II. Taxones nuevos. Brenesia 45-46: 33-50.

Rojas, A.F. 2001. Seis especies nuevas y dos nuevos registros de helechos (Pteridophyta) para Costa Rica. Rev. Biol. Trop. 49: 435-452.

Smith, A.R. 1993. Terpsichore, a new genus of Grammitidaceae (Pteridophyta). Novon 3: 478-489.

Smith, A.R., R.C. Moran \& L.E. Bishop. 1991. Lellingeria, a new genus of Grammitidaceae. Amer. Fern J. 81(3): 76-88. 


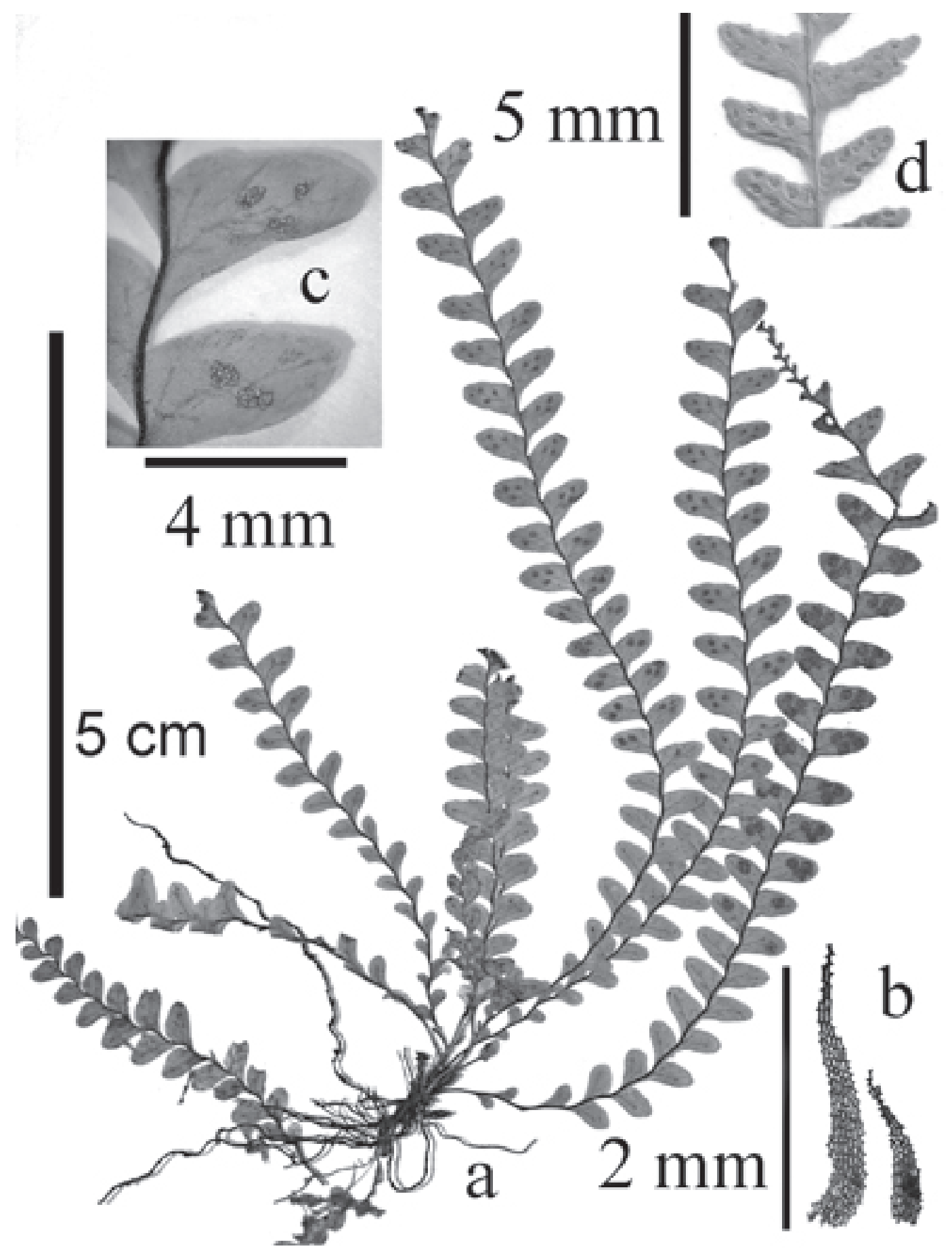

Fig. 1. Holotipo de Lellingeria pinnata A. Rojas (G. Herrera \& A. Chacón 2728, CR). a) Hábito. b) Escamas del rizoma. c) Sección de la lámina. Lellingeria phlegmaria (J. Sm.) A.R. Sm. d) Sección de la lámina. 


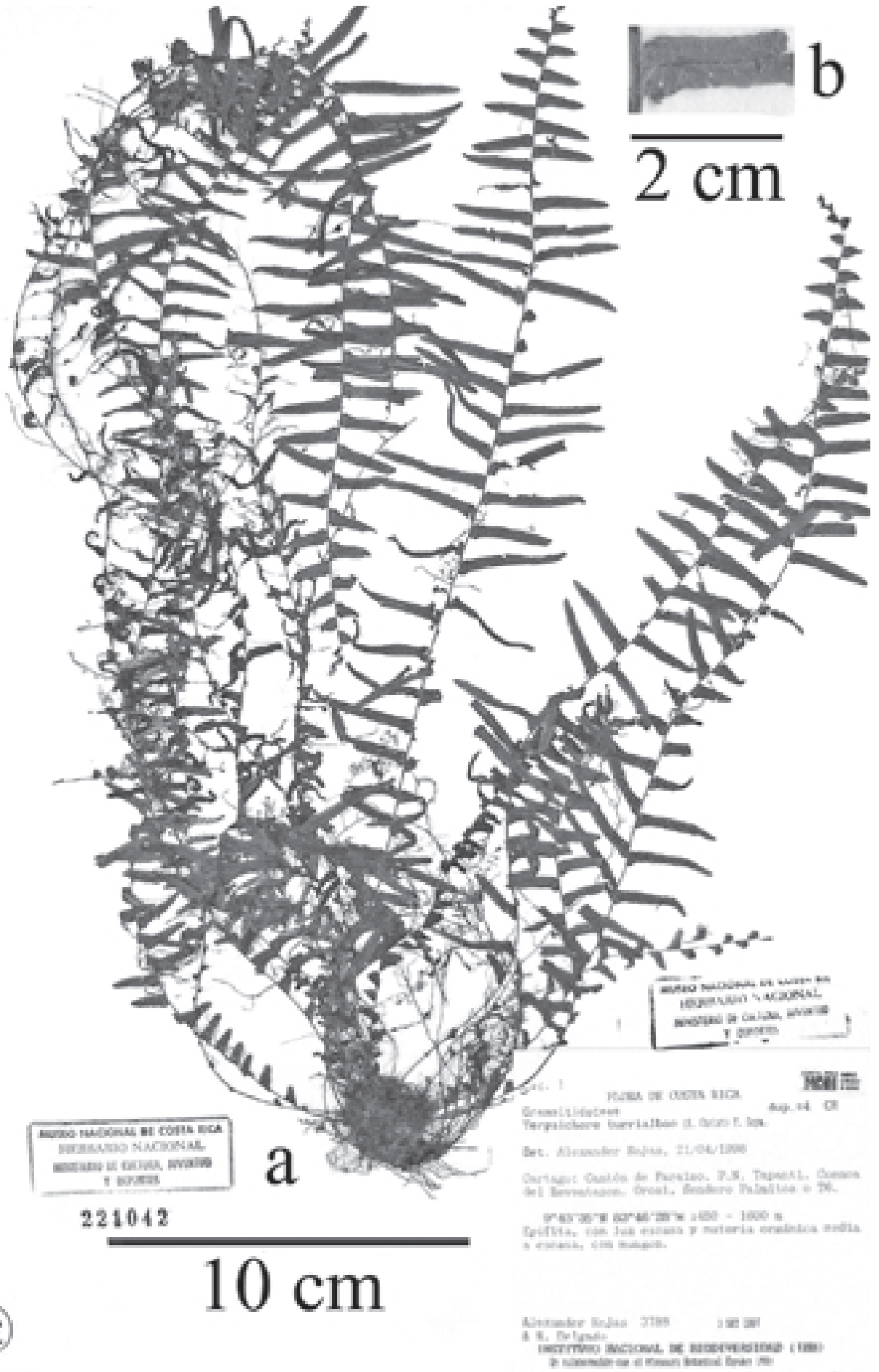

Fig. 2. Holotipo de Terpsichore glandulifera A. Rojas (A. Rojas \& R. Delgado 3788, CR). a) Hábito. b) Detalle de una pinna. 


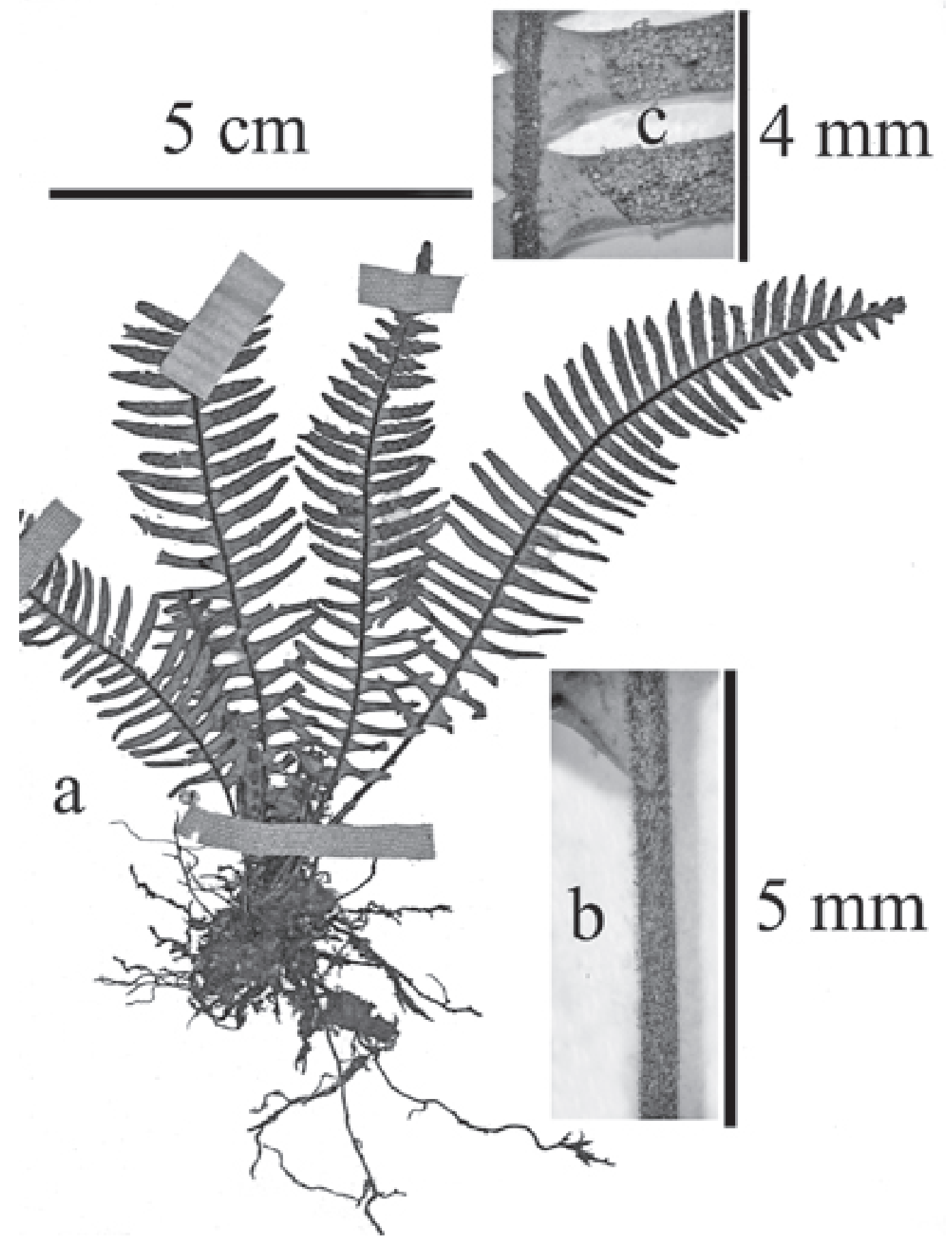

Fig. 3. Ejemplar representativo de Terpsichore pirrensis A.R. Sm. (D. Lellinger \& J. White 1089, CR). a) Hábito. b) Sección del pecíolo. c) Sección de la lámina. 